\title{
Avances de la legislación sobre Responsabilidad Social Empresaria en Argentina
}

\section{Development of Corporate Social Responsibility Legislation in Argentina}

\author{
Miguel BlazQuez* \\ María Florencia Peretti \\ MÓNICA Buraschi \\ Leonardo Sedevich Fons \\ Alfredo Mondino \\ Universidad Nacional de Córdoba, Argentina
}

Recibido el 2 de noviembre de 2009, aceptado el 11 de enero de 2010.

$\mathrm{N}^{\mathrm{o}}$ de clasificación JEL: M14

DOI: $10.5295 /$ cdg. $100182 \mathrm{mb}$

\section{Resumen:}

El objetivo del presente trabajo es reflejar las acciones legislativas que se han emprendido desde el estado Argentino en sus diferentes jurisdicciones en materia de responsabilidad social empresaria. A partir del mismo se pretende fijar posición desde la perspectiva de los autores sobre su escenario futuro.

Teniendo en cuenta que no existe una legislación integral de carácter nacional, se toman como ejemplo algunas legislaciones e iniciativas de alcance local. Para ello se analiza la Ley $N^{o} 2594$ de la Legislatura de la Ciudad Autónoma de Buenos Aires, la cual exige la presentación de un balance social empresario. Se analiza también la Ley Nacional $N^{o} 25877$ de Ordenamiento del Régimen Laboral en relación a la presentación de un Balance Social y las propuestas de modificaciones a la misma y además otro proyecto regulatorio en la materia que se originó en la Municipalidad de San Carlos de Bariloche.

\section{Abstract:}

The purpose of this paper is, on the one hand, to disclose the legislative actions that Argentine Government its different jurisdictions has undertaken about Corporate Social Responsibility in; and, on the other hand, to set the authors' point of view as regards its future development.

Considering the fact that there is no a comprehensive national legislation about this subject, some local laws and initiatives are taken as example. One of them is Act No. 2594 by Legislatura de la Ciudad Autónoma de Buenos Aires, which demands companies to publish entrepreneurial social reports. Another source is national Act No. 25877 and its modification proposals, which deals with Working Regime Order in relation to Social Report publications. Besides, another regulatory project about the topic from Municipalidad de San Carlos de Bariloche is taken into account.

Palabras clave:

Responsabilidad social empresaria, balance social, stakeholders, Argentina.

Key Words:

Corporate Social Responsibility, Social Report, Stakeholders, Argentina.

\footnotetext{
* Investigadores del Instituto de Administración de la Facultad de Ciencias Económicas de la Universidad Nacional de Córdoba, Argentina. Av. Valparaíso s/n - Ciudad Universitaria (5000) Córdoba-Argentina. mab@eco. unc.edu.ar/fperetti@eco.unc.edu.ar.
} 


\section{INTRODUCCIÓN}

Este trabajo forma parte de un proyecto de investigación desarrollado en el Instituto de Administración de la Facultad de Ciencias Económicas de la Universidad Nacional de Córdoba. Dicho proyecto, denominado "Los reportes de sustentabilidad en Argentina: vigencia y perspectivas", cuenta con el aval académico de la Secretaría de Ciencia y Tecnología de la UNC y el tema tratado en este artículo, responde a uno de los objetivos del mismo.

Aquí nos proponemos indagar sobre la legislación formulada desde los distintos estamentos del Estado Argentino en relación al concepto de responsabilidad social empresaria que se viene desarrollado en el ámbito privado. Teniendo en cuenta las diferentes interpretaciones que pueden derivarse de dicho concepto y que de hecho se reflejan indirectamente en las diferentes propuestas legislativas analizadas creemos conveniente desde nuestra perspectiva definir la responsabilidad social como "el compromiso de la empresa a contribuir al desarrollo sostenible, con la participación de sus grupos de interés, a fin de mejorar la calidad de vida de la sociedad en su conjunto" (CEADS, 2003); resaltando que el principal objetivo del desarrollo sostenible es "satisfacer las necesidades del presente sin poner en peligro la capacidad de las generaciones futuras para satisfacer sus propias necesidades". (GRI G3, 2006)

Teniendo en cuenta que no existe una legislación de carácter nacional se toman como ejemplo algunas legislaciones e iniciativas de alcance local. Para ello se analiza la Ley No 2594 de la Legislatura de la Ciudad Autónoma de Buenos Aires promulgada el 6 de Diciembre de 2007 que a la fecha no se ha puesto en funcionamiento, la cual exige la presentación de un balance social empresario, como así también otros intentos normativos que se encuentran a nivel de proyectos.

Debemos aclarar que se podrán encontrar términos como balance social, balance de responsabilidad social y ambiental o reportes no financieros para referirnos, en forma indistinta, a los instrumentos donde se plasman los conceptos sobre responsabilidad social o sustentabilidad organizacional. Aún reconociendo que conceptualmente estos términos asumen diferentes contenidos, los que fueron abordados acabadamente en el proyecto original de la investigación, su finalidad es muy semejante, ya que tienen el propósito de servir de soporte a la organización para formalizar ante la comunidad un comportamiento ético y responsable sobre la forma de gestionar su actividad.

De esta manera se tratará de abordar los siguientes interrogantes: ¿Cuáles son los avances, características y alcance de la legislación argentina en la materia? ¿Es necesario que el Estado promueva la responsabilidad social empresaria? 


\section{ANÁLISIS DE LA LEGISLACIÓN EXISTENTE}

\subsection{Ley $\mathbf{N}^{\circ} \mathbf{2 5 9 4}$ de Balance de Responsabilidad Social y Ambiental - Legislatura de la Ciudad Autónoma de Buenos Aires}

\section{A. Principales características}

La ley fue promulgada el 6 de Diciembre del 2007, no obstante ello hasta la fecha no se ha hecho efectiva. Han existido una serie de reclamos de los sectores involucrados y es por ello que se encuentra en un proceso de debate para su reglamentación definitiva.

Algunas de las características de la ley son:

- Presentación obligatoria del balance social para las empresas de más de 300 trabajadores.

- Opcional para el resto de las empresas, con beneficios de tipo financieros para quienes lo realicen.

- Presentación anual, con indicadores concretos, objetivos y mensurables.

- Se podrán tomar como guía los indicadores desarrollados por ETHOS (Brasil) o GRI.

- Firma por responsable idóneo.

- Publicación en la página web para acceso de todo interesado.

- Creación de un Comité de Enlace Intergubernamental (involucra a varios ministerios) para la evaluación y seguimiento tendientes a su mejor implementación.

- Otorgamiento de ciertos beneficios y/o prioridades de acceso para aquellas empresas, fundaciones, ONGs, organismos que voluntariamente presenten anualmente su balance social.

Se podría observar la legitimidad de establecer obligaciones diferenciales tomando como base la cantidad de trabajadores como indicador de su capacidad económica y financiera, ya que una gran empresa con alta tecnología pero poca mano de obra empleada podría quedar eximida, mientras que una PYME de servicios con gran cantidad de personal estaría obligada.

De igual manera también se podría observar que algunos de los indicadores relacionados con la responsabilidad social empresaria se encuentran ya legislados y que por lo tanto su cumplimiento es obligatorio, como por ejemplo los referidos a la legislación laboral que rigen para todo tipo de organización independientemente de su magnitud. En estos casos, el Estado estaría duplicando sus funciones y generando superposición de organismos públicos.

Pero independientemente de éstas y otras observaciones de genuino fundamento, no podemos dejar de observar algunos aspectos que hacen a una diferencia cualitativa entre la formalidad de una obligación adicional por parte del sector público a lo que frecuentemente se ven comprometidas las empresas, como por ejemplo el cumplimiento de una nueva ley laboral, legislación impositiva o de seguridad ambiental, y el alcance que adquiere la presentación formal de un balance social y ambiental con las particularidades que le otorga la presente ley. 
Una de estas características diferenciales se puede encontrar en su articulo décimo: “crea un registro de público acceso y disponible en página web del GCBA que enunciará las organizaciones inscriptas y los Balances de responsabilidad Social y Ambiental (BRSA) presentados. Luego de verificar que el contenido del BRSA se ajuste a lo establecido por la ley, la autoridad inscribirá a la organización en el registro, con renovación anual. En el registro también se incluirá un listado de empresas obligadas que no hayan dado cumplimiento con la presentación. La autoridad de aplicación también agrupará las organizaciones en función de presentación voluntaria u obligatoria y en función de los compromisos asumidos. Este parámetro de compromisos será relevante para asignar a los beneficiarios prioridad de acceso a los beneficios".

Para comprender el verdadero significado de este artículo, es necesario analizar por un lado la importancia que adquiere la información puesta a disposición a través de la tecnología informática y comunicacional en que cada persona desde diferentes lugares del mundo pueda acceder a la misma en el momento que lo desee y en este caso en particular tomar conocimiento del balance social y ambiental de cualquier empresa y/u organismo público que se encuentre alcanzado por la legislación en la Ciudad Autónoma de Buenos Aires. Desde otra perspectiva también se debe analizar que la comunidad organizada en función de sus diferentes intereses (muchos de ellos originados en un sentido altruista de compromiso ciudadano) exige cada vez más un comportamiento social del conjunto de las organizaciones. Así, ya sea en forma organizada a través de las múltiples y crecientes instituciones del sector no gubernamental (ONG) como también de los propios ciudadanos en el ejercicio de sus funciones, se demanda cada vez con mayor frecuencia información confiable y sistematizada del conjunto de las organizaciones que conviven en un determinado espacio social (como puede ser el municipio). De esta manera el balance social de las empresas y/o diferentes organismos se transforma en una carta de presentación institucional en el ámbito de la jurisdicción en la que operan, con el grado de confiabilidad que le otorga el ser supervisado por una institución pública (en este caso el municipio) sin perjuicio de la propia imagen de quien debe velar por su cumplimiento.

La segunda característica significativa está dada en la comprensión de que las organizaciones son entes en permanente cambio y desarrollo con una dinámica cada vez más creciente; es por ello que de la misma manera que se hace necesario cumplir con determinadas obligaciones en un momento dado (como quien saca una fotografía) también se hace necesario promover un desarrollo con alto contenido social. En este sentido la frase "en función de los compromisos asumidos" permite observar la película de una determinada organización, ya que los compromisos asumidos vienen acompañados de un plazo de ejecución al tiempo que se establecen nuevas obligaciones. Este ciclo modifica el entramado social de las organizaciones en búsqueda de complementariedad, más que de enfrentamiento y hostilidad como han demostrado en muchos casos los procesos históricos.

Otra particularidad significativa se encuentra en el artículo décimo al establecer "la constitución de un Comité de Enlace Intergubernamental con participación de los diferentes ministerios". Aquí se pone en evidencia que el tema del balance social no puede ser asignado a un área específica sino que es muy probable que comprenda a todas y a cada una de las áreas ya existentes, como así también otros aspectos que hoy se encuentran fuera de una jurisdicción especifica. Si bien no existe antecedente permanente de comité de enlace, 
las nuevas organizaciones se deben comenzar a encuadrar dentro de este nuevo funcionamiento, en correspondencia con la complejidad que caracteriza a la gestión pública.

De igual manera la diferenciación de empresas con obligatoriedad para aquellas de mayor magnitud, pone de relieve la importancia política que se le asigna al tema, a la vez que no compromete el patrimonio y la fuente de trabajo de organizaciones menores que pueden verse resentidas en la obligación de realizar un conjunto de acciones que originan un costo, en algunos casos, muy significativo. Simultáneamente, se inicia un proceso de cambio que seguramente exigirá realizar ajustes sucesivos y que por lo tanto es prudente no hacerlo en forma generalizada; esta flexibilidad legal está contemplada en la normativa para incorporar las modificaciones que se crean convenientes, al mencionar en su artículo 16 “...Asimismo participará de un Comité de Enlace Intergubernamental involucrando a los Ministerios de Ambiente y Espacio Público, Hacienda, Desarrollo Social y Planeamiento y Obras Públicas con el objeto de evaluar la marcha de la ley y las acciones tendientes a su mejor implementación”.

\section{B. Estado actual}

Si bien la ley fue publicada en el Boletín Oficial el 28 de enero del 2008 y se establecía la reglamentación dentro de 60 días el mismo se fue dilatando en el tiempo. Ante una nueva gestión de gobierno se estableció un Consejo Consultivo sobre Responsabilidad Social y Ambiental. La resolución 2840/MDEGC/08 tiene por objetivo institucionalizar un espacio de diálogo y participación con los distintos sectores de la sociedad cuyos representantes serán: las Asociaciones de la Sociedad Civil, ONGs promotoras de la RSE, organizaciones empresarias e instituciones educativas, interesadas en participar en el proceso consultivo para la reglamentación de la Ley.

Algunas de las observaciones que se plantearon son: "las directrices a nivel internacional se plantean como voluntarias, y que la imposición de parámetros en un sentido o en otro no beneficia la internalización de las normas por parte de la sociedad”; "...la norma dictada busca imponer una obligación para las empresas sin analizar ni apalancar las reales ventajas que significa para las organizaciones incorporar RSE." (Fabris, L; 2009); "Si la presentación de dicho balance fuera obligatorio, la implementación real de acciones en materia de RSE se reducirá a su mínima expresión. Dicha obligatoriedad no sólo implica importantes sobrecostos administrativos y de gestión para las empresas, sino que fundamentalmente, violenta el espíritu básico de la RSE' (Comunicarse, 2009).

Estas fundamentaciones no necesariamente se ajustan a la realidad mundial ya que existen muchos países que han avanzado sobre el tema como Francia, Australia, Inglaterra, Dinamarca y Noruega donde están haciendo obligatoria alguna forma de reporte que incluya aspectos ambientales y sociales. (Rsearch, 2009).

De igual manera la crisis mundial económica y financiera del 2009 ha generado la declaración de Amsterdam sobre la transparencia de información sobre la sustentabilidad: "El Consejo de la Global Reporting Initiative (GRI) declaramos que la poca transparencia que existe en el sistema actual de hacer informes empresariales ha fallado a sus grupos de interés. Aunque reconocemos que determinados gobiernos han demostrado su liderazgo en divulgación de información sobre sostenibilidad corporativa, hacemos un llamamiento 
a todos los gobiernos para que amplien y refuercen el sistema mundial de elaboración de memorias de sostenibilidad. En concreto, se deben volver a analizar las suposiciones acerca de la adecuación de la información voluntaria"; "Las raíces de la actual crisis económica podrían haberse moderado con un sistema de transparencia y rendición de cuentas basado en el ejercicio de la debida diligencia y la información publica”.

Debemos recordar que en nuestro país no existe una normativa nacional que imponga la presentación de un informe público y periódico de contenido social y ambiental que complemente a la presentación del balance económico que presenta el estado de resultados. A continuación se analizará un antecedente a nivel nacional sobre la presentación de un "Balance Social", pero la confidencialidad de los datos condiciona su carácter público y por lo tanto también la posibilidad de ser considerado como tal, por entender que la importancia de toda presentación formal sobre la gestión no financiera de cualquier tipo de organización radica justamente en su carácter público.

\subsection{Otros antecedentes legales en la Argentina}

Entre los antecedentes de legislación sobre responsabilidad social empresaria en nuestro país se destacan tres iniciativas, tanto a nivel nacional como municipal: la Ley Nacional de Ordenamiento del Régimen Laboral No 25877 del 18 de marzo de 2004; el Proyecto de Ley Responsabilidad Social Empresaria de Leguizamón y Capitanich tendiente a modificar algunos artículos de dicha ley y el Proyecto de la Municipalidad de San Carlos de Bariloche.

La Ley No 25877 en el capítulo IV del Título “Derecho Colectivo del Trabajo" establece que las empresas que posean un número de trabajadores superior a 300 deberán elaborar, anualmente, un balance social, que se presentará al Ministerio de Trabajo, Empleo y Seguridad Social, "la que será considerada estrictamente confidencial". Como se mencionó anteriormente, este carácter confidencial limita los alcances del reporte a presentar.

En su articulo 26 se requiere la siguiente información:

“a) Balance general anual, cuenta de ganancias y pérdidas, notas complementarias, cuadros anexos y memoria del ejercicio.

b) Estado y evolución económica y financiera de la empresa y del mercado en que actúa.

c) Incidencia del costo laboral.

d) Evolución de la masa salarial promedio. Su distribución según niveles y categorías.

e) Evolución de la dotación del personal y distribución del tiempo de trabajo.

f) Rotación del personal por edad y sexo.

g) Capacitación.

h) Personal efectivizado. Régimen de pasantías y prácticas rentadas.

j) Estadísticas sobre accidentes de trabajo y enfermedades inculpables.

k) Terciarizaciones y subcontrataciones efectuadas.

l) Programas de innovación tecnológica y organizacional que impacten sobre la plantilla de personal o puedan involucrar modificación de condiciones de trabajo." 
Como se puede observar los contenidos y características del Balance Social requerido por esta ley son totalmente diferentes a los expresados en el proyecto de la Municipalidad de Buenos Aires. En este caso la información solicitada se encuentra expresamente indicada y está orientada exclusivamente a la problemática de los recursos humanos, su incidencia económica, capacitación y accidentes de trabajo, entre otros, asegurando en cierta manera su reserva como información estadística y la posibilidad de generar análisis de tipo macroeconómicos y/o sectoriales. Por el contrario el Balance Social que requiere la Municipalidad de Buenos Aires, tiene un amplio sentido informativo y su contenido queda enmarcado dentro de las guías de sustentabilidad que existen en la actualidad.

Cabe aclarar que si bien la Ley $\mathrm{N}^{\circ} 25877$ se encuentra vigente, el capítulo referido al Balance Social no fue reglamentado, por lo que no se cumplimenta en la práctica.

\section{PROYECTOS DE LEY}

\subsection{Ley sobre Responsabilidad Social Empresaria en el Senado de la Nación. Dirección Publicaciones (S-3735/05) De Leguizamón y Capitanich}

El presente proyecto de ley de RSE se relaciona con la Ley No 25877. En sus distintos artículos modifica algunas disposiciones vigentes, como por ejemplo extendiendo la obligación de presentación del balance social a las empresas contratistas del sector público y a aquéllas que acudan al financiamiento de los mercados financieros organizados, aunque tengan menos de 300 trabajadores.

En su articulo tercero define la "Responsabilidad Social Empresaria, a la adopción por parte de la empresa de un compromiso de participar como ciudadana, contribuyendo a aliviar los problemas de la comunidad donde está inserta asumiendo una conducta ética Es una filosofía corporativa, conjunto de políticas, prácticas y programas adoptada por todos los integrantes de la empresa para actuar en beneficio de los trabajadores, su público de relacionamiento, la comunidad y el ambiente que perdura a lo largo del tiempo" y al "Balance social de la empresa, al documento que recoge los resultados cuantitativos y cualitativos del cumplimiento de la responsabilidad social de la empresa y permite evaluar su desempeño en términos de activos y pasivos sociales durante un período determinado".

De igual manera propone la derogación de privacidad del balance social que "se hará público con el balance y memorias anuales con igual tratamiento, transparencia y difusión que el balance económico del ejercicio" que a su vez deberá ser "comunicado por la representación de la empresa a la representación sindical de los trabajadores con un plazo de 30 (TREINTA) días de anterioridad para su examen, a efectos de consulta previo a la aprobación definitiva".

Establece un conjunto de indicadores mínimos sin perjuicios de otros que la empresa crea conveniente referidos a los ítems: a) Recursos Humanos b) Clientes y Proveedores c) Ambiente d) Comunidad. Quienes acrediten tal situación podrán "utilizar públicamente el distintivo de 'Socialmente Responsable' con la identificación de la marca de la entidad que haya extendido la certificación”.

Por último los balances sociales de las empresas descriptas en este inciso deberán ser validados por una auditoría social externa. 


\subsection{Proyecto en la Municipalidad de San Carlos de Bariloche}

Paralelamente, también ha tomado estado público la existencia de proyectos sobre RSE en otras ciudades como la de San Carlos de Bariloche. En el Proyecto de Ordenanza $\mathrm{N}^{\circ}$ 291-CM-08 se establece que dichas prácticas no deben ser obligatorias, pero teniendo en cuenta que un la función primordial del estado es promover el bienestar general, se hace necesario promover sin exigir la RSE. Para ello se propone un registro para aquellas empresas que se identifican con el tema, teniendo en cuenta que ello significa "una ventaja comparativa y un elemento sustancial para distinguirse de sus competidoras" y que "el establecimiento de los indicadores permitirá la objetiva valoración y evaluación de las condiciones de equidad y sustentabilidad social", como se indica en los Fundamentos que anteceden a la Ordenanza. El otorgamiento por parte del Municipio de un certificado de RSE servirá además para que las empresas reciban el acompañamiento por parte del Estado Municipal a través de programas especiales o planes de financiamiento.

Estos son algunos ejemplos vinculados a las acciones concretas que puede llevar adelante la empresa para cumplir con las exigencias del certificado de RSE: Predicar con el ejemplo (publicitar la filosofía de manejo social responsable de la organización, liderar el cambio de actitud en la comunidad); participar de las actividades comunitarias (los eventos culturales, sociales, conmemorativos o deportivos que son valiosos para la sociedad); instalar valores mediante la implementación de acciones concretas como premiar a los mejores alumnos, a los líderes sociales, y la manifestación concreta contra cualquier tipo de discriminación; desarrollar alianzas estratégicas con proveedores, clientes, dirigentes comunales y la sociedad civil, que apunten a mejorar la calidad de vida comunitaria; promover la asociatividad, la participación y el involucramiento social.

Por último la Municipalidad publicará semestralmente en medios de comunicación las empresas de la ciudad que cumplieron con dichos criterios ya que serán auditadas por las autoridades de aplicación o por entidades externas independientes si es que la empresa así lo desea.

\section{REFLEXIONES}

Debemos tener presente que la propia Constitución Nacional en su artículo 41 menciona "Todos los habitantes gozan del derecho a un ambiente sano, equilibrado, apto para el desarrollo humano y para que las actividades productivas satisfagan las necesidades presentes sin comprometer las de las generaciones futuras; y tienen el deber de preservarlo." y "Corresponde a la Nación dictar las normas que contengan los presupuestos mínimos de protección, y a las provincias, las necesarias para complementarlas, sin que aquellas alteren las jurisdicciones locales...”. Dentro de este enfoque existe un documento Base de la Secretaría de Desarrollo Sustentable y Política Ambiental en el que se describen los lineamientos de la Estrategia Nacional de Desarrollo Sustentable, cuyos objetivos son entre otros:

- "Involucrar a la población en el proceso de desarrollo sustentable

- Promover y facilitar el flujo de información y conocimientos a todos los niveles 
- Incorporar la dimensión ambiental en los ámbitos micro y macroeconómicos

- Desarrollar y aplicar a nivel nacional criterios e indicadores de desarrollo sustentable"

El desarrollo de la responsabilidad social empresaria no responde a una expresión unilateral de un sector de la sociedad preocupado en un momento de su historia por mejorar su propia gestión ante la comunidad, sino a una conjunción de múltiples factores económicos, sociales, tecnológicos y de funciones entre los sectores de una sociedad organizada que ha dado origen a una demanda creciente por parte de la comunidad con diferentes grados de expresión hacia el sector público y el sector privado en temas relacionados con su calidad de vida desde lo económico, cultural y ambiental.

Escapa a este trabajo profundizar el tema como así también simplificar el análisis en presuponer que la RSE es solamente la expresión de un requerimiento social externo, sin comprender que también es una respuesta de quienes tienen altas responsabilidades sociales. Lo que pareciera estar fuera del debate es la necesidad de generar y promover nuevos estilos de gestión tendientes a disminuir las asimetrías sociales crecientes, ya no sólo entre países sino también entre los sectores de una misma comunidad, a la vez que se pretende consolidar el entramado social que permita generar un ambiente de viabilidad y estabilidad para el desarrollo de las diferentes actividades. Como menciona Klisksberg $(2005 ; 46)$ "Globalización también es el crecimiento paradójico de un mundo afuera del globo. La marginación y la exclusión, que aquí llamo la asimetría de la vulnerabilidad y exclusión compartida, nos lleva a las cuestiones de poder y justicia que son cuestiones éticas fundamentales. El momento ético ocurre cuando se conoce esta vulnerabilidad asimétrica, y se asume como tarea personal y colectiva." En una reciente publicación de este mismo autor en forma conjunta con el Nobel de Economia Amartya Sen $(2009,198)$ ratifican “... este problema en América Latina no es sólo de políticas públicas, sino de compromiso social empresario: Hay que dar el salto de la filantropía social a la responsabilidad social empresaria: buenas condiciones y medio ambiente de trabajo para los empleados, precios razonables y cuidado de los recursos naturales".

El mundo se ha globalizado, las fronteras se ven relativizadas, la información y la comunicación han dejado de ser una limitación. Empiezan a generarse demandas de estabilidad y sostenibilidad del ambiente, de cumplimiento de regímenes laborales, de igualdad de género, de transparencia de capitales y de una mirada de conjunto sobre la equidad, transparencia y ética de la gestiones empresarias.

La complejidad es un signo del mundo moderno y es también un desafío desde lo social, económico y científico encontrar las respuestas a los nuevos problemas que se caracterizan por interacciones múltiples cuyas relaciones de causa y efecto han dejado de ser lineales. Se exigen nuevas lógicas de pensamiento, nuevas formas de gestionar y de evaluar y nuevas formas de administrar y organizar. Desde esta apretada síntesis el tema de la Responsabilidad Social Empresaria no puede ser abordado con exclusividad por un solo sector de la sociedad sino que requiere el concurso de toda la sociedad para lograr una respuesta efectiva y mancomunada.

Pensar en una responsabilidad social empresaria limitada únicamente a quienes deben llevarla a cabo no sólo es vaciarla de contenido en un proceso histórico signado por los nuevos fenómenos de crisis económica, sino también promover una fragmentación de ro- 
les institucionales que condiciona su propia esencia. La cooperación es uno de los pilares sobre los que se construye la RSE, y para ello exige el compromiso del conjunto de la sociedad y el fomento de quienes tienen responsabilidad de estado. Este trabajo mancomunado no debe cercenar la iniciativa privada ni las demandas sociales para un crecimiento sostenido, sino simplemente establecer el marco de referencia mínimo en el rol de estado que promueva y estimule un comportamiento generalizado para aquéllos que no logran comprender los nuevos desafíos.

Es por ello que será también función del estado garantizar la difusión de información pública, confiable y sistematizada de las acciones realizadas operando como un nexo de legitimidad entre comunidad y empresa. Para ello consideramos que no es necesario un nuevo organismo, como ha ocurrido en Francia donde se ha creado un ministerio de la sustentabilidad, sino que basta con integrar en comités los ya existentes para que la suma de experiencia de partes (sinergia de sistemas) también pueda llenar de contenido a este nuevo desafío. Como bien menciona Lozano Soler (2007) “Estamos en una organización de redes donde el liderazgo, compromiso y convicción tanto en el ámbito empresarial como en el político y social son claves para impulsar una competividad responsable y sostenible" y luego agrega: "La necesidad de gobernanza de los procesos de globalización ya no involucra solamente a los gobiernos y a las instituciones políticas; la alianza entre los diferentes actores se ha hecho indispensable, así como su corresponsabilizaciòn, sobre todo en lo referente al establecimientos de acuerdos básicos compartidos".

\section{CONCLUSIONES}

Como todo proceso social, los cambios son consecuencia de un conjunto de diferentes dimensiones en las que la legislación es tan solo una de ellos, dentro de la complejidad que caracteriza el mundo moderno. Los conceptos de responsabilidad social empresaria, en sus diferentes denominaciones e instrumentos que la gestionan es un ejemplo más de un proceso social positivo en cuanto a calidad de vida pero en un contexto local de asimetrías crecientes en la fragmentación y exclusión social. En este sentido el estado debe mediar en la cohesión y equidad junto a la información y participación sin excluir ningún tiempo ni espacio.

Temas como el presente son también parte de la agenda oficial y estamos convencidos que si bien los intentos analizados aún son muy embrionarios ponen de manifiesto una tendencia que debiera generalizarse no sólo por nosotros mismos sino también por las generaciones futuras, si es que anhelamos un mundo sustentable.

\section{REFERENCIAS BIBLIOGRÁFICAS}

Comunicarse. "Buenos Aires debate modificaciones en su ley de RSE" [en línea] Buenos Aires, Marzo de 2009. Disponible en: http://www.comunicarseweb.com.ar/biblioteca/noticias-09/0201. html [Consulta: 2 de octubre de 2009]

FABRIS, L. La responsabilidad social empresaria y la ley 2594 de la Ciudad Autónoma de Buenos Aires. [en línea] Buenos Aires. Erreius.com. Disponible en: http://www.codigor.com.ar/brsa.htm [Consulta: 2 de octubre de 2009] 
Global Reporting Initiative: Declaración de Ámsterdam sobre Transparencia e Información. [en línea]. Ámsterdam, 2009. Disponible en: http://www.globalreporting.org/NewsEventsPress/ PressResources/SpanishTranslationAmsterdamDeclaration.htm [Consulta: 15 de setiembre de 2009]

Instituto Boliviano de Comercio Exterior: Responsabilidad Social Empresarial: Hacia el Triple Sello, Comercio Exterior, $N^{\circ}$ 174, Julio 2009. Disponible en: http://www.ibce.org.bo/publicaciones/ comext descarga.asp?id=116 [Consulta: 12 de agosto de 2009]

KLIKSBERG, B. (comp.) La agenda ética pendiente de América Latina, Bs. As., Editorial Fondo de Cultura Económica de Argentina. S.A., 1 ${ }^{\text {a }}$ Edición. 2005.

KLIKSBERG, B. y Sen, A.: Primero la gente, España, Editorial Deusto, 1ª Edición. 2009

Ley $N^{\circ} 2594$ de Balance de Responsabilidad Social y Ambiental -. Publicada en el Boletín Oficial de la Ciudad Autónoma de Buenos Aires No 2859 del 28 de enero de 2008. Disponible en: http:// boletinoficial.buenosaires.gov.ar/areas/leg_tecnica/boletines/20080128.htm\#1. [Consulta: 2 de octubre de 2009]

Ley $N^{\circ} 25877$ de Ordenamiento del Régimen Laboral del 18 de marzo de 2004. Publicada en el Boletín Oficial de la República Argentina No 30364 del 19 de marzo de 2004. Disponible en: http://www.infoleg.gov.ar/infolegInternet/verNorma.do?id=93595. [Consulta: 29 de setiembre de 2009]

LOZANO SOLER, J. M.: Promoción pública de la responsabilidad social empresarial, Ekonomiaz, ISSN 0213-3865, $\mathrm{N}^{\circ}$ 65. 2007. Disponible en: http://dialnet.unirioja.es/servlet/ articulo?codigo $=2533614$ [Consulta: 14 de setiembre de 2009]

Proyecto de Ley de María L. Leguizamón.- Jorge M. Capitanich.- Secretaría Parlamentaria Dirección Publicaciones (S-3735/05) Senado de la Nación Argentina. Disponible en: http://comisiones. senado.gov.ar/web/proyectos/verExpe.php?origen $=$ S\&tipo=PL\&numexp=3735/05\&nr o_comision $=\& t$ Consulta $=3$. [Consulta: 29 de setiembre de 2009]

Proyecto de Ordenanza N $N^{\circ}$ 291-CM-08 de la Municipalidad de San Carlos de Bariloche, Argentina. Disponible en: http://www.concejobariloche.gov.ar/index.php?option=com_content\&task=view \&id=3902\&Itemid=472 [Consulta: 2 de octubre de 2009].

Research. Publicación de Tendencias y Actualidad de RSE. Madrid, Editorial Forética, $\mathrm{N}^{\circ} 1$, Julio 2009. http://www.foretica.es/es/index.asp?MP=33\&MS=85\&MN=1\&TR=A\&IDR=1\&iddocum ento=621. [Consulta: 15 de setiembre de 2009]

Secretaría de Desarrollo Sustentable y Política Ambiental: Documento Base de la Estrategia Nacional de Desarrollo Sustentable (ENDS)- Diciembre 2001. Disponible en: http://www.ambiente. gov.ar/archivos/web/DCSyLD/File/ends.pdf [Consulta: 7 de setiembre de 2009] 
